\title{
Bending of flexible round plates
}

\author{
Adash Yuldashev, Shamshod Pirmatov ${ }^{*}$, Sherzod Bekchanov, and Hamidullakhon Axralov
}

Tashkent State Technical University, Tashkent, Uzbekistan

\begin{abstract}
In this paper, schemes for constructing solutions to boundary value problems for static calculation of flexible circular plates with the nonlinear theory of Lyava and Volmyr are presented. From the equations of the equilibrium system of the plates, given in curvilinear coordinates, the system of equilibrium equations for flexible round plates is obtained. Substituting the expressions for the efforts and shearing forces and introducing dimensionless quantities, we obtain a system of quasilinear quantities in displacements.

To develop an automated system for static calculation of flexible round plates, we use central finite-difference schemes that approximate derivatives with second-order accuracy, we obtain a system of quasilinear algebraic equations. To test the constructed automatic system for static calculation, the difference equations are reduced to vector form. An implicit iterative process combined with the Gaussian elimination method is applied to the solution of the system of equations. When calculating iterative processes, it continues until the above conditions are met. After determining the required functions by the finite difference method, we calculate the calculated values. Using the obtained numerical results, we will construct their graphs.
\end{abstract}

\section{Introduction}

From the literature reviewed, it can be seen that most of the problems on flexible circular plates are solved in the Fepple-Karmana formulation, which is a special case of Lyava [1]. The constructed algorithms are not economical about their implementation on the computer. Therefore, the construction of an automated system for the complete calculation of flexible round plates with a given degree of accuracy becomes an urgent issue.

The problem of creating an automated system was first posed in the monograph by V.K. Kabulov [2]. The algorithmization problem is solved in four stages. At the first stage, depending on the geometric characteristics of the object and the physical properties of the material, the design scheme of this model is selected. The second stage is associated with the derivation of the original differential equations in the corresponding boundary and initial conditions. The choice of a computational algorithm and the numerical solution of the obtained solutions constitutes the third stage of research. The fourth stage ends with the analysis of the obtained numerical results described by the stress-strain state of the structure under consideration.

\footnotetext{
*Corresponding author: shamshod@rambler.ru
} 
In particular, in [9], the algorithms for calculating specific structures - beams, plates and conical shells - are considered.

This work in the formulation of Love formulated boundary value problems of flexible circular plates in displacements. The corresponding system of two nonlinear partial differential equations is reduced to a system of two quasilinear differential equations.

The solution of a system of difference equations with different boundary conditions is reduced to the solution of systems of quasilinear equations.

\section{Methods}

The choice of a computational algorithm and the numerical solution of the formulated boundary value problems constitute the main stage of algorithmization

This paper addresses the following issues:

1) Construction of a unified computational scheme for solving boundary value problems of static calculation of flexible round plates using the nonlinear Lyava theory;

2) Development of an automated system for static calculation of flexible round plates;

3) Approbation of the built automated system;

4) Study of the nature of the convergence of the applied numerical methods.

Let us use the well-known equations of equilibrium of the plate in an arbitrary curvilinear coordinate system $[1,3,18,19]$.

$$
\left.\begin{array}{c}
\frac{\partial}{\partial \alpha}\left(B \bar{T}_{1}\right)-\frac{\partial}{\partial \beta}\left(A \bar{S}_{2}\right)-\left(r_{1} B \bar{S}_{1}+r_{2} A \bar{T}_{2}\right)+\left(q B \bar{Q}_{1}+q_{2} A \bar{Q}_{2}\right)+A B X=0 \\
\frac{\partial}{\partial \alpha}\left(B \bar{S}_{1}\right)+\frac{\partial}{\partial \beta}\left(A \bar{T}_{2}\right)-\left(p_{1} B \bar{Q}_{1}+p_{2} A \bar{Q}_{2}\right)+r_{1} B \bar{T}_{1}-r_{2} A \bar{S}_{2}+A B Y=0 \\
\frac{\partial}{\partial \alpha}\left(B \bar{Q}_{1}\right)+\frac{\partial}{\partial \beta}\left(A \bar{Q}_{2}\right)-\left(q_{1} B \bar{T}_{1}-q_{2} A \bar{S}_{2}\right)+p\left(B \bar{S}_{1}+p_{2} \bar{A}_{2}\right)+A B Z=0
\end{array}\right\}
$$

where: $\alpha, \beta, \gamma$ are curvilinear coordinates; $A, B$ are the coefficients of the first coordinate form; $\bar{T}_{1}, \bar{T}_{2}, \bar{S}_{1}$ and $\bar{S}_{2}$ are components of membrane forces; $\bar{Q}_{1}, \bar{Q}_{2}$ are shearing forces; $r_{1}, r_{2}, q_{1}, q_{2}, p_{1}, p_{2}$ are surface curvature; $X, Y, Z$ are volumetric forces.

Equations of equilibrium of flexible circular plates under the action of axisymmetric loads are derived from this system.

$$
\left.\begin{array}{l}
\frac{d}{d \bar{r}}\left(\bar{T}_{1} \bar{r}\right)-\bar{T}_{2}-\bar{r} \bar{Q} \frac{d^{2} w}{d \bar{r}^{2}}=0 \\
\frac{d}{d \bar{r}}\left(\bar{Q}_{\bar{r}}^{-}\right)-\bar{r}_{1} \frac{d^{2} \bar{w}}{d \bar{r}^{2}}+\bar{T}_{2} \frac{d \bar{w}}{d \bar{r}}+\bar{r} \bar{q}=0
\end{array}\right\}
$$

Here

$$
\bar{T}_{1}=\frac{12}{h^{2}} D\left(\bar{\varepsilon}_{11}+\mu \bar{\varepsilon}_{22}\right), \bar{T}_{2}=\frac{12}{h^{2}} D\left(\bar{\varepsilon}_{11}+\mu \bar{\varepsilon}_{22}\right)
$$




$$
\begin{aligned}
& \bar{Q}=-D\left(\frac{d^{3} \bar{w}}{d \bar{r}^{3}}+\frac{1}{\bar{r}} \frac{d^{2} w}{d \bar{r}^{2}}-\frac{1}{r^{2}} \frac{d \bar{w}}{d \bar{r}}\right) \\
& \bar{\varepsilon}_{11}=\frac{d \bar{u}}{d \bar{r}}+\frac{1}{2}\left(\frac{\partial \bar{w}}{\partial \bar{r}}\right)^{2}, \bar{\varepsilon}_{22}=\frac{\bar{u}}{\bar{r}}
\end{aligned}
$$

For round plates, this is

$$
\begin{aligned}
& h_{1}=1, h_{2}=\frac{1}{r}, A=1, B=\bar{r}, p_{1}=0, q_{1}=-\frac{d^{2} \bar{w}}{d \bar{r}^{2}}, r_{1}=\frac{d^{2} \bar{v}}{d \bar{r}^{-2}}, p_{2}=\frac{d \bar{w}}{d \bar{r}} \\
& q_{2}=0, r_{2}=1, s=s_{1}=-s_{2}=0, Q_{2}=0, Q_{1}=-Q, X=0, Y=0, Z=\bar{q}
\end{aligned}
$$

Substituting expressions for efforts (2) and for shearing forces (3) $[3,8,13]$ into (1) and introducing the following without dimensional quantities $[9,10]$.

$$
r=\frac{\bar{r}}{a}, u=\frac{\bar{u}}{h}, w=\frac{\bar{w}}{h}, \delta=\frac{a}{h}
$$

we obtain the equilibrium equations in displacements

$$
\left.\begin{array}{l}
-a_{1} \frac{d^{2} u}{d r^{2}}-a_{2} \frac{d u}{d r}+a_{3} u-a_{4} \frac{d^{2} w}{d r^{2}}-a_{5} \frac{\partial w}{\partial r}=0 \\
b_{1} \frac{d^{4} w}{d r^{4}}+b_{2} \frac{d^{3} w}{d r^{3}}-b_{3} \frac{d^{2} w}{d r^{2}}+b_{4} \frac{d w}{d r}=\beta
\end{array}\right\}
$$

where

$$
\begin{aligned}
& a_{1}=12 \delta^{2}, a_{2}=\frac{12 \delta^{2}}{r}, a_{3}=\frac{12 \delta^{2}}{r^{2}}, a_{4}=\frac{1}{\delta} \frac{d^{3} w}{d r^{3}}+\frac{1}{r \delta} \frac{d^{2} w}{d r^{2}}+\left(12 \delta-\frac{1}{r^{2} \delta}\right) \frac{d w}{d r}, \\
& a_{5}=\frac{6(1-\mu)}{r} \delta \frac{d w}{d r}, b_{1}=1, b_{2}=\frac{2}{r}, b_{3}=\frac{1}{r^{2}}+12 \delta\left[\frac{d u}{d r}+\frac{1}{2 \delta}\left(\frac{d w}{d r}\right)^{2}+\mu \frac{u}{r}\right], \\
& b_{4}=\frac{1}{r^{3}}-\frac{12}{r} \delta\left[\frac{u}{r}+\mu \frac{d u}{d r}+\mu \frac{1}{2 \delta}\left(\frac{d w}{d r}\right)^{2}\right], \beta=q_{0} q, q_{0}=\frac{12\left(1-\mu^{2}\right)}{E} \delta^{4}, q=q(r) .
\end{aligned}
$$

System (7) is solved at $0 \leq r \leq 1$ - for solid and at $r_{0} \leq r \leq 1$ - for an annular circular plate and with the following boundary conditions

$$
\left.T_{0} \delta u_{r}\right|_{\Gamma}=0,\left.M_{v} \delta \frac{\partial w}{\partial v}\right|_{\Gamma}=0,\left.R \delta u\right|_{\Gamma}=0
$$

Equations of equilibrium of flexible round plates (7) for the given boundary conditions can also be solved using the method of meshes [4, 5, 14].

Let's introduce a grid: 


$$
w_{h}=\left\{\begin{array}{l}
r_{i}=i h-\text { solid round plate }\left(0 \leq r_{i} \leq 1\right) \\
\mathrm{r}_{\mathrm{i}}=\mathrm{r}_{0}+\mathrm{ih}\left(1-\mathrm{r}_{0}\right)-\text { annular circular plate }\left(\mathrm{r}_{0} \leq r_{i} \leq 1\right) .
\end{array},\right.
$$

step along coordinate $h=\frac{1}{N}, X_{i}=\left\{U_{i}, W_{i}\right\}$.

Using the central difference formulas that approximate the derivatives with a second order accuracy $[4,6]$, instead of equations (7), we obtain the following system of quasilinear algebraic equations $[7,9]$ :

$$
A_{i} X_{i-2}+B_{i} X_{i-2}+C_{i} X_{i}+D_{i} X_{i+1}+E_{i} X_{i+2}=q
$$

where

$$
\begin{aligned}
& A_{i}=\left(\begin{array}{cc}
0 & 0 \\
0 & a_{22}
\end{array}\right), \mathrm{B}_{\mathrm{i}}=\left(\begin{array}{cc}
b_{11} & b_{12} \\
0 & b_{22}
\end{array}\right), C_{i}=\left(\begin{array}{cc}
c_{11} & c_{12} \\
0 & c_{22}
\end{array}\right), \\
& \left.D_{i}=\left(\begin{array}{cc}
d_{11} & d_{12} \\
0 & d_{22}
\end{array}\right), E_{i}=\left(\begin{array}{cc}
0 & 0 \\
0 & e_{22}
\end{array}\right), g_{i}=\left(\begin{array}{l}
0 \\
\beta
\end{array}\right)\right\} \\
& b_{11}=a_{1} N^{2}-a_{2} \frac{N}{2}, b_{12}=a_{4} N^{2}-a_{5} \frac{N}{2}, c_{11}=2 a_{1} N^{2}+a_{3}, c_{12}=2 a_{4} N^{2} \text {, } \\
& d_{11}=a_{1} N^{2}+a_{2} \frac{N}{2}, \\
& d_{12}=a_{4} N^{2}+a_{5} \frac{N}{2}, a_{22}=b_{1} N^{4}-b_{2} \frac{N^{3}}{2}, e_{22}=b_{1} N^{4}+b_{2} \frac{N^{3}}{2}, \\
& b_{22}=4 b_{1} N^{4}-b_{2} N^{3}+b_{3} N^{2}+b_{4} \frac{N}{2}, c_{22}=6 b_{1} N^{4}+2 b_{3} N^{2} \text {, } \\
& d_{22}=4 b_{1} N^{4}+b_{2} N^{3}+b_{3} N^{2}-b_{4} \frac{N}{2}, a_{1}=12 \delta^{2}, a_{2}=12 \frac{\delta^{2}}{r_{i}}, a_{3}=12 \frac{\delta^{2}}{r_{i}}, \\
& a_{4}=\frac{N^{3}}{2 \delta}\left(w_{i+2}-2 w_{i+1}+2 w_{i-1}-w_{i-2}\right)+\frac{N^{2}}{r_{i} \delta}\left(w_{i+1}-2 w_{i}+w_{i-1}\right)+\left(12 \delta-\frac{1}{r_{i}^{2} \delta} w_{i+1}-w_{i-1}\right) \frac{N}{2}, \\
& b_{1}=1, b_{2}=\frac{2}{r_{i}}, b_{3}=\frac{1}{r_{i}^{2}}+12 \delta\left[\frac{N}{2}\left(u_{i+1}-u_{i-1}\right)+\frac{N^{2}}{8 \delta}\left(w_{i+1}-w_{i-1}\right)^{2}+\mu \frac{u_{i}}{r_{i}}\right], \\
& b_{4}=\frac{1}{r_{i}^{3}}-\frac{12 \delta}{r_{i}}\left\{\frac{u_{i}}{r_{i}}+\mu \frac{N}{2}\left[\left(u_{i+1}-u_{i-1}\right)+\mu \frac{N^{2}}{8 \delta}\left(w_{i+1}-w_{i-1}\right)^{2}\right]\right\} \text {, }
\end{aligned}
$$

Let us consider some different boundary conditions for flexible circular plates under uniformly distributed loads.

For a solid round plate hinged on the contour

$$
\left.\begin{array}{l}
\left.u\right|_{r=0}=0,\left.w^{\prime}\right|_{r=0}=0,\left.w^{\prime \prime \prime}\right|_{r=0}=0, \\
\left.u\right|_{r=1}=0,\left.w\right|_{r=1}=0,\left.w^{\prime}\right|_{r=1}=0
\end{array}\right\}
$$


We get from the first, fourth and fifth (9)

$$
U_{0}=0, U_{N}=0, W_{N}=0
$$

Applying the central difference formulas with the second order of approximation [5] to the second, third, and sixth conditions (9), we find $[6,15,16,20]$.

$$
\left.\begin{array}{l}
w_{0}=\frac{4}{3} w_{1}-\frac{1}{3} w_{2} \\
w_{-1}=\frac{4}{9} w_{1}+\frac{8}{9} w_{2}-\frac{1}{3} w_{3}, w_{N+1}=w_{N-1}
\end{array}\right\}
$$

In vector form, conditions (10) and (11) are written as follows:

$$
\left.\begin{array}{l}
E X_{0}=A_{0} X_{1}+B_{0} X_{2}, \\
E_{-1} X_{-1}=A_{-1} X_{1}+B_{-1} X_{2}+C_{-1} X_{3}
\end{array}\right\}
$$

and

$$
X_{N}=0, E_{N} X_{N+1}=E_{N} X_{N-1}
$$

Substituting (12) and (13) into the system of equations (8), we obtain the system of quasilinear algebraic equations

$$
M X=\tilde{b}
$$

where

$$
\begin{aligned}
& M=\left\{\begin{array}{c}
\bar{C}_{1}, \bar{D}_{1}, \bar{E}_{1} \\
\bar{B}_{2}, \bar{C}_{2}, D_{2} E_{2} \\
A_{3} B_{3} C_{3} D_{3} E_{3} \\
A_{4} B_{4} C_{4} D_{4} E_{4} \\
\ldots \ldots \ldots \ldots \ldots \ldots \ldots \ldots \ldots \\
\ldots \\
\ldots
\end{array}\right\} \\
& \bar{C}_{1}=C_{1}+A_{1} A_{-1}+B_{1} A_{0}, \bar{D}_{1}=D_{1}+A_{1} \bar{B}_{-1}+B_{1} B_{0}, \\
& \bar{E}_{1}=E_{1}+A_{1} C_{-1}, \bar{B}_{2}=A_{2} A_{0}+B_{2} \text {, } \\
& \bar{C}_{2}=C_{2}+A_{2} B_{0}, \bar{C}_{N-1}=C_{N-1}+E_{N-1} E_{N},
\end{aligned}
$$




$$
\begin{aligned}
& A_{0}=\left(\begin{array}{ll}
0 & 0 \\
0 & \frac{4}{3}
\end{array}\right), B_{0}=\left(\begin{array}{cc}
0 & 0 \\
0 & -\frac{1}{3}
\end{array}\right)=C_{-1}, E_{N}=\left(\begin{array}{ll}
0 & 0 \\
0 & 1
\end{array}\right), \\
& A_{-1}=\left(\begin{array}{ll}
0 & 0 \\
0 & \frac{4}{9}
\end{array}\right), B_{-1}=\left(\begin{array}{ll}
0 & 0 \\
0 & \frac{8}{9}
\end{array}\right) .
\end{aligned}
$$

To solve the system of quasilinear algebraic equations (14), an implicit iterative process is applied in combination with the Gaussian elimination method, whose equations have the following form $[4,6]$ :

$$
X_{i}=\alpha_{i} X_{i+1}+\beta_{i} X_{i+2}+\gamma_{i}
$$

where,

$$
\left.\begin{array}{l}
\alpha_{i}=-\Theta_{i}\left(D_{i}+\bar{\theta}_{i} \beta_{i-1}\right), \beta_{i}=-\Theta_{i} E_{i}, \\
\gamma_{i}=\Theta_{i}\left[\tilde{b}_{i}-\left(\bar{\theta}_{i} \gamma_{i-1}+A_{i} \gamma_{i-2}\right)\right], \\
\Theta_{i}=\left(c_{i}+A_{i} \beta_{i-2}+\bar{\Theta}_{i} \alpha_{i-1}\right), \bar{\Theta}_{i}=A_{i} \alpha_{i-2}+\beta_{i}
\end{array}\right\},
$$

The iterative process in calculating (19) continues until the condition

$$
\left|X_{i}^{(j+1)}-X_{i}^{(j)}\right|-\varepsilon \leq 0
$$

where $\varepsilon$ is the accuracy of the solution.

Using the forward sweep formulas (16) with $i=N$, we find the values of the last unknown vector $X_{N}$ and $X_{N-1}$. Then, using formula (15), we determine the values of the grid function $X_{i}(i=\overline{1, N-2})$.

After determining the required function $X_{i}$ by the finite difference method, the calculated values are calculated using the following formulas:

$$
\begin{gathered}
\varepsilon_{11}=\frac{N}{2 \delta}\left[u_{i+1}-u_{i-1}+\frac{N}{4 \delta}\left(w_{i+1}-w_{i-1}\right)^{2}\right], \varepsilon_{22}=\frac{u_{i}}{\delta r_{i}}, \chi_{11}=\frac{N^{2}}{\delta^{2}}\left(w_{i+1}-2 w_{i}+w_{i-1}\right), \\
\chi_{22}=\frac{N^{2}}{2 \delta^{2}}\left(w_{i+1}-w_{i-1}\right), e_{11}^{k}=\varepsilon_{11}+(-1)^{k} \frac{1}{2} \chi_{11}, e_{22}^{k}=\varepsilon_{22}+(-1)^{k} \frac{1}{2} \chi_{22},(k=1,2) \\
T_{1}=\varepsilon_{11}+\mu \varepsilon_{22}, T_{2}=\varepsilon_{22}+\mu \varepsilon_{11}, M_{1}=-\left(\chi_{11}+\mu \chi_{22}\right), M_{2}=-\left(\chi_{22}-\mu \chi_{11}\right), \\
Q=-\frac{1}{\delta^{3}}\left\{\frac{N^{3}}{2}\left(w_{i+2}-2 w_{i+1}+2 w_{i-1}-w_{i-2}\right)+\frac{N^{2}}{r_{i}}\left(w_{i+1}-2 w_{i}+w_{i-1}\right)-\frac{N}{2 r_{i}^{2}}\left(w_{i+1}-w_{i-1}\right)\right\},
\end{gathered}
$$




$$
\sigma_{1}^{k}=\sigma_{1}^{M}+(-1)^{k} \sigma_{1}^{u}, \sigma_{2}^{k}=\sigma_{2}+(-1)^{k} \sigma_{2}^{u},(k=1,2)
$$

\section{Results and Discussion}

A solid round plate hinged along the contour. The calculation was carried out for the following values of geometric and mechanical characteristics: $\delta=\frac{a}{h}=40 ; \beta=12\left(1-\mu^{2}\right) \delta^{4} \frac{q}{E}=12.8$ and 38.4. $\varepsilon=10^{-5}, \mu=0,3$.

The calculations were performed at $N=10,20,40$. The main calculation results are summarized in the graph $(1.1,1.2)$. $W$ (Figure 1), $\sigma_{1}^{1}, \sigma_{1}^{2}, \sigma_{2}^{1}, \sigma_{2}^{2}$ (Figure $1 \mathrm{c}, \mathrm{d}, \mathrm{e}, \mathrm{f}$ ), $M_{1}, M_{2}$ (Figure $2 \mathrm{c}, \mathrm{d}$ ), $T_{1}, T_{2}$ (Figure 1 and 2) reach their extreme values in the center plates $\sigma_{1}^{2}, Q$ (Figure 2 e) - on the contour, and $U$ (Figure 1) at $r=0,5$.
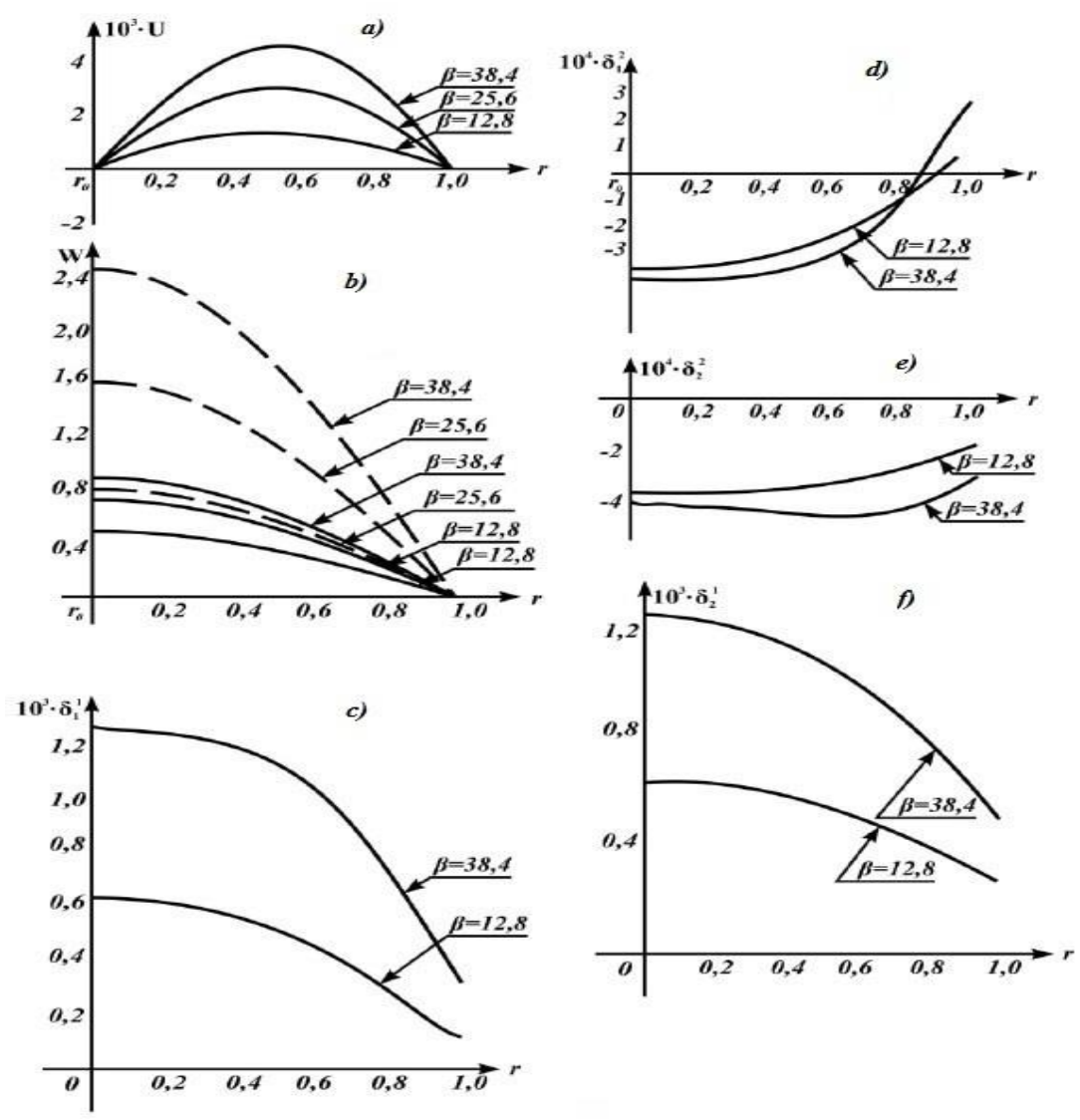

Fig. 1. a) $u$-is radial displacement, b) $w$-is deflection, c), d) $\sigma_{1}^{1}, \sigma_{1}^{2}-$ are radial total stresses, f) $\sigma_{2}^{1}, \sigma_{2}^{2}$ - are tangential total stresses. 

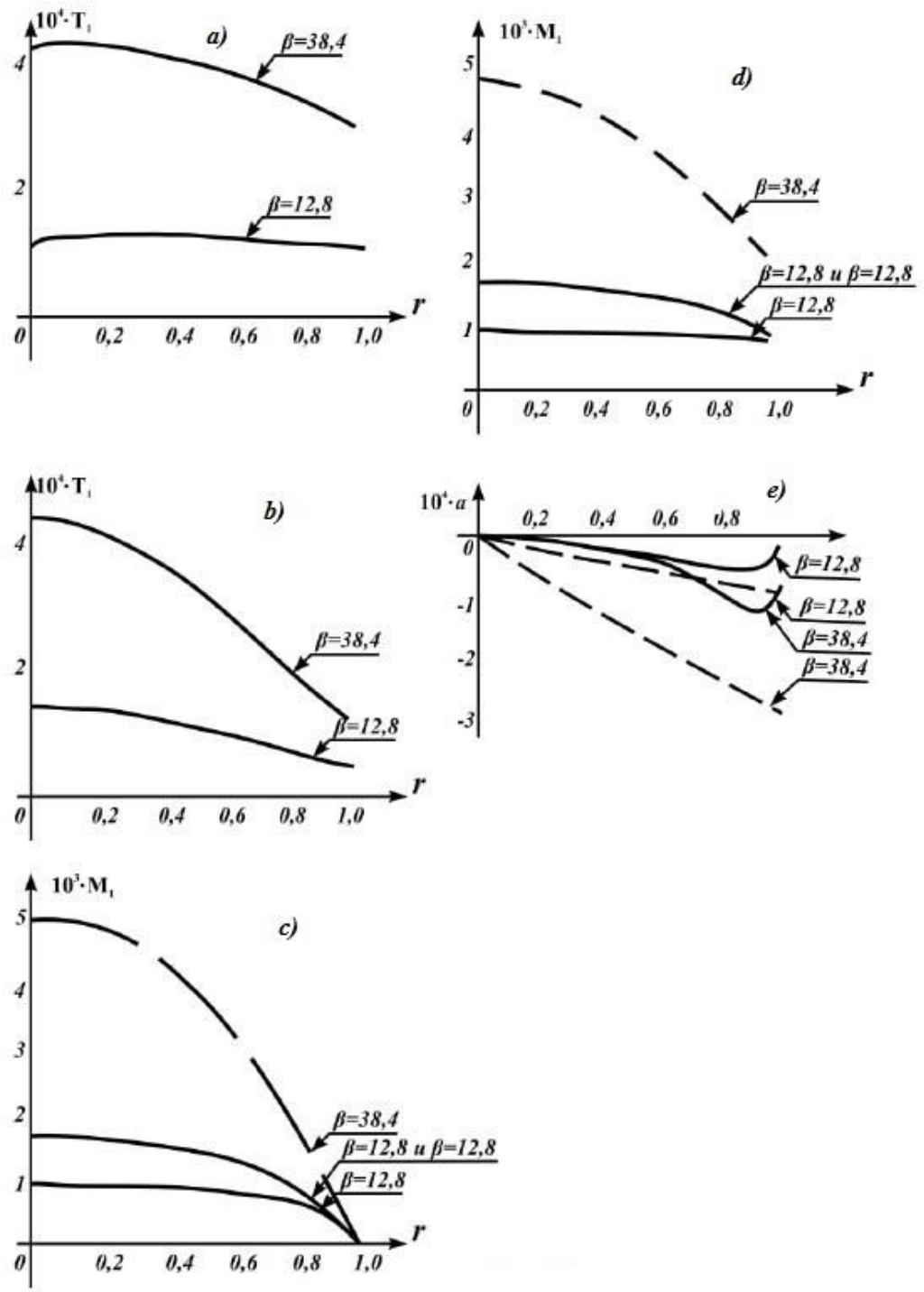

Fig. 2. a), b) $T_{1}, T_{2}-$ are radial and tangential force, c), d) $M_{1}, M_{2}-$ are bending moments, e) $Q-$ is cutting force.

The difference in the values of the deflection and moment of the plates in the nonlinear formulation of the problem with respect to the linear and total stresses relative to the bending in the linear formulation in the center is, respectively, at $\beta=12.8 k \approx 60 ; 68.31 \%$ and at $\beta=38.4 k=170 ; 381 \%$. At $12.8 ; 38.4$, it changes sign, corresponding, in points $r=0.885 ; 0.844$. The table with $\delta=40, N=20, \varepsilon=10^{-6}, \mu=0,3$ shows the deflection values $w_{\lambda}(0), w_{H \lambda}(0)$ according to Lyava's theory, $w^{I}(0)$ - according to Kornishin, $w^{I I}(0)$ - according to Ueyav. 
Table 1.

\begin{tabular}{|c|c|c|c|c|}
\hline$\beta$ & $w_{\lambda}(0)$ & $w_{H \lambda}(0)$ & $w^{I}(0)$ & $w^{I I}(0)$ \\
\hline 24.9 & 0.38918 & 0.362133 & 0.363100 & 0.362871 \\
56.0196 & 0.875585 & 0.692801 & 0.694 & 0.693743 \\
83.9748 & 1.31242 & 0.904869 & 0.906 & 0.90582 \\
125.9076 & 1.96790 & 1.142414 & 1.150 & 1.14863 \\
189.916 & 2.96806 & 1.407727 & 1.41 & 1.40914 \\
\hline
\end{tabular}

It can be seen from the table that the value of the deflection of the plates $w_{H \lambda}(0)$ according to Lyava's theory, $w^{I}(0)$-Kornishin, $w^{I I}(0)$ - according to Ueyav coincide in two decimal places, and according to the other signs, according to Lyava's theory, the results are more underestimated than according to the theory of Kornishin and Ueyav.

\section{Conclusions}

The main results of the work are summarized as follows:

1. A unified computational scheme for solving boundary value problems of static calculation of flexible round plates by the method of finite differences has been constructed. In the formulation of boundary value problems in displacements, the nonlinear theory of Volmira was used [3].

2. An automated system for complete static calculation of flexible circular plates with arbitrary boundary conditions has been built. The system is based on standard education programs and solutions to large systems of nonlinear algebraic equations. Calculating the calculated values and printing the results.

3. The character of convergence of the finite difference method and implicit iterative processes of solving systems of nonlinear algebraic equations depending on the intensity of the external load was investigated. It was found that the deflections obtained by M.S. Kornishen [8] turned out to be overestimated. The convergence rate of the iterative process does not depend on the number of nodes.

4. It was found that with an increase in the degree of nonlinearity of the problem, the amplitude of the calculated bending values decreases.

\section{References}

1. A. Lyav. Mathematical theory of elasticity. M.-L.ONTI, USSR, p. 674, (1935)

2. Kabulov V.K. Algorithmization in the theory of elasticity and deformation theory of plasticity. Tash. Publishing house "Fan" UzSSR. (1966)

3. Volmir A.S. Flexible plates and sheaths. M.: Gostekhizdat, p. 420, (1956)

4. Demidovich B.P., Maron I.A. Numerical methods of analysis. M.: fizmatgiz, p. 367, (1962)

5. Berezin I.S., Zhidkov N.P. Calculation methods. T.1.2.M

6. Demidovich B.P., Maron I.A. Numerical methods of analysis. Edited by B.P. Demidovich, M.Fizmatgiz, (1962)

7. Yuldashev A., Pirmatov Sh.T., Minarova N. Equations of equilibrium of flexible round plates. Austrian J. Technical and Natural Sciences, № 3-4, pp.32-35, (2015)

8. Kornishin M.S. Some questions of the application of the finite difference method for solving boundary value problems in the theory of plates. "Applied Mechanics", 9, № 36 (1963)

9. Buriev T. Application of computers to the calculation of elliptical plates. Abstract of candidate dissertation, Tashkent, publishing house "Fan", UzSSR, (1965) 
10. A. Yuldashev, Sh.T. Pirmatov. Algorithmization of solving dynamic edge problems of the theory of flexible rectangular plate. Bulletin of the Tomsk State University "Mathematics and Mechanics". № 66. pp. 143-157. (2020), DOI: 10.17223 / 19988621/66/12.

11. Vyachkin E.S., Kaledin V.O., Reshetnikova E.V., Vyachkina A.E. Development of a mathematical model of static deformation of a sandwich structure with pressed layers. Bulletin of the Tomsk State University "Mathematics and Mechanics". 2018 № 55. pp. 72-83. DOI: 10.17223 / 19988621/55/7.

12. Korobeinikov S.N. Nonlinear deformation of solids. Novosibirsk; Publishing house SORAN. p. 262, (2000)

13. Robotov Yu. N. Mechanics of a deformable body M: Nauka, p. 712, (1988)

14. Berikkhanova G.E., Zhumagulov B.T., Kanguzhin B.E. Matematicheskaya model' kolebaniy paketa pryamougol'nykh plastin s uchetom tochechnykh svyazey.Tomsk State University Journal of Mathematics and Mechanics. 1 (9). pp. 72-86, (2010)

15. Yuldashev A., Bekchanov Sh.E., Akhralov Kh.Z. Algorithmic Solution of Dynamic Boundary Problems in the Flexible Circular Plates Theory. Journal Technical science and innovation. №4, (2020)

16. Yuldashev A., E.A.Nikolayeva, Sh.T.Pirmatov. Statistical calculation of flexibel circular plates. Technical science and innovation. №2/, (2020)

17. Bate K.Yu. Finite Element Methods. Moscow: Fizmatgiz, p. 1024, (2010)

18. Timoshchenko S.P., Voinovsky-Krieger S. Plates and shells, M., Fizmatgiz, (1963)

19. Timoshchenko S.P. Shell plates, M., Gostekhizdat, (1948)

20. Volmira A.S., Theory of flexible round plates, Trans. from Chinese, (1957) 